\title{
Utilização de planejamento experimental no estudo para imobilização de lodo galvânico em cerâmica vermelha para minimização de impactos ambientais
}

\section{(Use of experimental design in the study of galvanic sludge immobilization in red ceramic for environmental impact minimization)}

\author{
R. D. C. da Rocha, H. E. Zorel Jr., T. Lando \\ Universidade Tecnológica Federal do Paraná, Via do Conhecimento, km 1, Pato Branco, PR, Brasil 85503-390 \\ raqueldcr@utfpr.edu.br
}

\begin{abstract}
Resumo
O trabalho proposto tem como objetivo avaliar por meio de planejamento estatístico a incorporação de um resíduo de galvanoplastia (lodo com metais altamente poluidores, como o cromo) em cerâmica vermelha, para a redução em seu armazenamento e a diminuição de sua contaminação ao meio ambiente e ao homem. Para isso, o lodo galvânico e a argila utilizada foram caracterizados quanto à densidade, caracterização visual, pH, umidade, matéria orgânica total, quantidade de cromo presente, espectroscopia de infravermelho e análise térmica (TG/DTA). Para avaliação do processo de produção do cerâmico foi realizado um planejamento fatorial $2^{3}$ (dois níveis e pontos centrais) em que as variáveis independentes foram concentração de lodo na massa, temperatura de secagem e temperatura de queima. As variáveis dependentes foram absorção de água, lixiviação do cromo, solubilidade de resíduos e retração linear, conforme seus limites estabelecidos. Analisando os resultados obtidos nas caracterizações dos corpos de prova, pôde-se identificar, após a verificação junto às normas vigentes, que houve duas concentrações de lodo na mistura dentro da maioria destas conformidades (5 e 15\%), com exceção do teste de solubilização, onde todos apresentaram valores acima do limite permitido. Por meio dos resultados obtidos, verificou-se a possibilidade de desenvolver produtos de cerâmica aditivados com lodo galvânico, porém nas faixas das variáveis independentes estudadas não foi possível a inertização do cromo.
\end{abstract}

Palavras-chave: metal pesado, resíduo galvânico, argila, cromo.

\begin{abstract}
The proposed study aims to evaluate by means of statistical design, the incorporation of galvanic sludge (with highly polluting metals, such as chromium) in red ceramic, in order to reduce its storage and contamination to the environment and the mankind. The galvanic sludge and clay used were characterized by density measurement, visual characteristics, $p H$, moisture, total organic matter, amount of chromium, infrared spectroscopy and thermal analysis (TG/DTA). For evaluation of the ceramic production process it was performed a $2^{3}$ factorial design (two levels and central points) where the independent variables were sludge concentration in the mass, drying temperature and firing temperature. The dependent variables were water absorption, chromium leaching, waste solubility and linear shrinkage, according to the established limits. Analyzing the obtained results in the characterization of the samples, it was possible to identify after checking with the current regulations, that there were two sludge concentrations in the mix which fit most of the compliances (5 and 15\%), except for the solution test, in which all samples showed results above the allowed limit. The achieved results showed the possibility of developing ceramic products added with galvanic sludge, but in the ranges of independent variables studied, it was not possible the chromium inertization.
\end{abstract}

Keywords: heavy metal, galvanic waste, clay, chromium.

\section{INTRODUÇÃO}

A atividade industrial possui geração de capital recorrendo aos mais diversos processos e tecnologias para a obtenção dos produtos desejados. Porém, esses processos geram grandes quantidades de resíduos que são, em muitos casos, de natureza ambientalmente perigosa, como é o caso da indústria galvânica, que gera lodo com metais altamente poluidores, como o cromo. Este resíduo, muitas vezes, por não ter destinação adequada, acumula-se no ambiente, tornando-se potencial causador de desastres ambientais. Ele deve ser minimizado de uma maneira economicamente viável ou armazenado. Na indústria galvânica, o principal processo é o tratamento de superfícies em que ocorre a formação de uma camada de revestimento, por meio da imersão de uma peça em uma solução contendo íon que se deseja depositar sobre a superfície sob o efeito de um diferencial de potencial. Esse processo tem a finalidade de conferir proteção contra corrosão, aumentar a dureza e a condutividade, além de possibilitar um aumento da vida útil da peça [1]. Entretanto, 
a galvanoplastia traz um ônus junto com seus benefícios, um processo crítico no que diz respeito à emissão de poluentes no ambiente, tanto no aspecto de grandes volumes de descartes, como também pelas suas características químicas, as quais são prejudiciais ao meio ambiente e aos seres humanos se descartados inadequadamente.

O lodo galvânico contém em média $63 \%$ de água e em sua composição estão presentes compostos químicos em diferentes formas, como hidróxidos e sais dos metais. Além de carbonatos e sulfatos devido ao processo de neutralização [2]. É considerado um resíduo - classe I (perigoso). Resíduos desta classe, devido às suas características de inflamabilidade, corrosividade, reatividade, toxicidade e patogenicidade, podem apresentar riscos à saúde pública [3], o que significa que podem apresentar efeitos adversos ao meio ambiente e à saúde humana quando manuseados ou dispostos de forma inadequada. Esse resíduo galvânico, sendo considerado perigoso, exige tipos de tratamentos diferenciados e disposições tecnicamente adequadas. Quando se trata de avaliar os impactos ambientais causados pelas atividades industriais, bem como a busca de soluções através de tecnologias para o tratamento dos resíduos gerados, torna-se necessária uma avaliação da destinação final destes resíduos [4].

Com o crescimento da exigência de implantação de políticas ambientais, as indústrias estão cada vez mais interessadas em maneiras de reduzir os resíduos gerados, bem como em desenvolver meios viáveis para o reaproveitamento e estabilização destes, para a disposição final de forma segura. A incorporação em cerâmicos é um tratamento que está sendo estudado para resíduos perigosos, pois o processo pode imobilizar esses resíduos, evitando assim a lixiviação e solubilização deste para o ambiente. O setor de construção civil vem mostrando um interesse na utilização de resíduos industriais no desenvolvimento de novos materiais, muitas vezes com melhores propriedades mecânicas, elétricas e térmicas. Estudos estão sendo realizados com o objetivo de desenvolver técnicas e tecnologias limpas e principalmente viáveis para a reutilização desses rejeitos. Vários autores têm demonstrado que esse processo pode ser utilizado para reciclar resíduos diferentes, tais como resíduos radioativos [5], cinzas do processo de incineração [6-8], resíduos de galvanização [9], resíduos ricos em ferro provenientes de processos hidrometalúrgicos [10] e lodo de tratamento de água [11]. Dessa forma, o trabalho proposto teve como objetivo avaliar por meio de planejamento estatístico a incorporação do resíduo de galvanoplastia em cerâmica vermelha com o intuito de minimizar a contaminação ao meio ambiente e ao homem.

\section{MATERIAIS E MÉTODOS}

O lodo galvânico e a argila utilizada na pesquisa foram coletados em indústrias do Sudoeste do Paraná. As amostras foram caracterizadas quanto aos seguintes parâmetros: densidade, $\mathrm{pH}$, umidade, matéria orgânica total, quantidade de cromo presente, espectroscopia de infravermelho e análise térmica. A densidade do lodo (Equação A) foi obtida por meio do método do picnômetro e o $\mathrm{pH}$ foi determinado por pHmetro (Q400AS Quimis).

$$
\mathrm{D}=\frac{\mathrm{D}_{\mathrm{ag}} \cdot\left(\mathrm{P}_{\mathrm{S}}-\mathrm{P}\right)}{\left(\mathrm{P}_{\mathrm{S}}-\mathrm{P}\right)-\left(\mathrm{P}_{\mathrm{sa}}-\mathrm{P}_{\mathrm{a}}\right)}
$$

em que, D é a densidade do material ( $\left(\mathrm{g} . \mathrm{L}^{-1}\right)$, $\mathrm{P}$ é a massa do picnômetro vazio $(\mathrm{g}), \mathrm{P}_{\mathrm{s}}$ massa da amostra $(\mathrm{g}), \mathrm{P}_{\mathrm{a}}$ massa do picnômetro com água, $\mathrm{P}_{\mathrm{sa}}^{\mathrm{s}}$ massa do picnômetro com água e amostra (massas medidas com balança analítica, AG200) e $\mathrm{D}_{\mathrm{ag}}$ é a densidade da água na temperatura da determinação. A porcentagem da umidade, matéria orgânica total e cinzas foram baseadas em metodologia padrões [12] e determinadas pelas Equações B, C e D, respectivamente:

$$
\begin{gathered}
\mathrm{U}=\frac{\left(\mathrm{P}_{2}-\mathrm{P}_{3}\right) \cdot 100}{\left(\mathrm{P}_{2}-\mathrm{P}_{1}\right)} \\
\mathrm{MO}=\frac{\left(\mathrm{P}_{3}-\mathrm{P}_{4}\right) \cdot 100}{\left(\mathrm{P}_{3}-\mathrm{P}_{1}\right)} \\
\mathrm{C}=100-\mathrm{MO}
\end{gathered}
$$

em que, U é a umidade da amostra (\%), MO porcentagem de matéria orgânica na amostra (\%), C porcentagem de cinzas na amostra (\%), $\mathrm{P}_{1}$ massa da cápsula tarada $(\mathrm{g}), \mathrm{P}_{2}$ massa da cápsula com amostra $(\mathrm{g}), \mathrm{P}_{3}$ massa da amostra $(\mathrm{g})$ após $24 \mathrm{~h}$ a $110{ }^{\circ} \mathrm{C}$ em estufa (Fanem 502/3A) e $\mathrm{P}_{4}$ massa da amostra após calcinação em mufla (Quimis) a $550{ }^{\circ} \mathrm{C}$. Para a análise térmica (análise termogravimétrica simultânea com análise térmica diferencial, TG/DTA) utilizou-se o equipamento Shimadzu, TA-50, sob atmosfera de nitrogênio de $20 \mathrm{~mL} \cdot \mathrm{min}^{-1}$, taxa de aquecimento de $10^{\circ} \mathrm{C} \cdot \mathrm{min}^{-1}$ e portaamostra de $\alpha$-alumina. A análise de infravermelho (IVTF) foi realizada no espectrômetro Biorad FTIR. A determinação do cromo foi realizada pelo método colorimétrico da difenilcarbazida [13].

Para avaliar a incorporação do logo galvânico na argila foi realizado um planejamento fatorial $2^{3}$ (dois níveis e pontos centrais), conforme Tabela I. As variáveis independentes foram concentração de lodo na massa, temperatura

Tabela I - Níveis das variáveis independentes: porcentagem de lodo na mistura, temperatura de secagem e temperatura de queima.

[Table I - Levels of independent variables: percentage of sludge in mixture, drying temperature and firing temperature.]

\begin{tabular}{cccc}
\hline Variável & $\begin{array}{c}\text { Nível } \\
(-1)\end{array}$ & $\begin{array}{c}\text { Nível } \\
(+1)\end{array}$ & $\begin{array}{c}\text { Ponto } \\
\text { central }(0)\end{array}$ \\
\hline $\mathrm{X}_{1}: \%$ de lodo & 5 & 25 & 15 \\
$\mathrm{X}_{2}:$ T. secagem $\left({ }^{\circ} \mathrm{C}\right)$ & 50 & 110 & 80 \\
$\mathrm{X}_{3}:$ T. queima $\left({ }^{\circ} \mathrm{C}\right)$ & 900 & 1100 & 1000 \\
\hline
\end{tabular}


Tabela II - Códigos de identificação dos corpos de prova.

[Table II - Identification codes of the specimens.]

\begin{tabular}{cccccccccc}
\hline Identificação & $1 \mathrm{AB}$ & $2 \mathrm{AB}$ & $3 \mathrm{AB}$ & $4 \mathrm{AB}$ & $1 \mathrm{CD}$ & $2 \mathrm{CD}$ & $3 \mathrm{CD}$ & $4 \mathrm{CD}$ & $1 \mathrm{E}$ \\
\hline Lodo $(\%)$ & 5 & 5 & 25 & 25 & 5 & 5 & 25 & 25 & 15 \\
T. secagem $\left({ }^{\circ} \mathrm{C}\right)$ & 50 & 50 & 50 & 50 & 110 & 110 & 110 & 110 & 80 \\
T. queima $\left({ }^{\circ} \mathrm{C}\right)$ & 900 & 1100 & 900 & 1100 & 900 & 1100 & 900 & 1100 & 1000 \\
\hline
\end{tabular}

de secagem e temperatura de queima. As variáveis dependentes foram absorção de água (AA), lixiviação do cromo (LC), solubilidade de resíduos (SR) e retração linear (RL). Na confecção dos corpos de prova com dimensões de $2 \mathrm{~cm}$ de altura por $5 \mathrm{~cm}$ de diâmetro, o lodo foi: i) secado a $105{ }^{\circ} \mathrm{C}$ até massa constante; ii) misturado (moinho de bola Cienlab) conforme a porcentagem estabelecida no planejamento experimental; iii) adicionado água (10\%) até obter massa homogênea e plástica (corpo de prova de 38 g); e iv) moldado em matriz metálica $(5 \mathrm{~cm} \mathrm{x} 12 \mathrm{~cm})$ por prensagem (20 MPa, prensa hidráulica universal Amsler). Após a prensagem, os corpos de prova foram identificados (Tabela II), secados em estufa (Fanem 502/3A) por 24 $\mathrm{h}$ e queimados em mufla (Quimis), como estipulado no planejamento fatorial.

$\mathrm{O}$ índice de absorção de água (AA) de cada corpo de prova foi determinado pela Equação E [14]. Para o método, os corpos de prova foram imersos em recipiente com água fervente durante $2 \mathrm{~h}$, resfriados até temperatura ambiente (submersos em água), e em seguida foi retirado o excesso de água com pano úmido e as massas foram medidas.

$$
\mathrm{AA}(\%)=\frac{\left(\mathrm{m}_{\mathrm{U}}-\mathrm{m}_{\mathrm{s}}\right)}{\mathrm{m}_{\mathrm{s}}} .100
$$

em que, $m_{u}$ e $m_{s}$ representam a massa úmida e a massa seca de cada corpo de prova, respectivamente, expressas em gramas. Para a metodologia de lixiviação [15], os corpos de prova foram moídos (moinho de martelo, Tecnal 330) até granulometria de $9,5 \mathrm{~mm}$ e adicionados $(10 \mathrm{~g}) \mathrm{em} 160 \mathrm{~mL}$ de água destilada. As misturas foram agitadas (30 rpm, MA085, Marconi) por $24 \mathrm{~h}$ e filtradas em membrana de $0,45 \mu \mathrm{m}$. Nos períodos de 15,45 e $105 \mathrm{~min}$, o $\mathrm{pH}$ da solução foi ajustado até $5,0( \pm 0,2)$ com solução de ácido acético $0,5 \mathrm{~N}$. No teste de solubilização [16] foram utilizados $20 \mathrm{~g}$ de cada corpo de prova (granulometria: $9,5 \mathrm{~mm}$ ) em $80 \mathrm{~mL}$ de água destilada. A extração foi realizada sob agitação $(30 \mathrm{rpm})$ por $5 \mathrm{~min}$, seguida de período de descanso de 7 dias; por fim, a mistura foi filtrada $(0,45 \mu \mathrm{m})$. Para verificação da retração linear [17] procederam-se medições geométricas dos corpos de prova, considerando o diâmetro e altura de cada peça, logo após a sua moldagem e após a queima. As medições foram realizadas com um paquímetro. A retração linear, RL, foi calculada por:

$$
\mathrm{RL}=\frac{\mathrm{L}_{\mathrm{i}}-\mathrm{L}_{\mathrm{f}}}{\mathrm{L}_{\mathrm{f}}} .100
$$

em que, $\mathrm{L}_{\mathrm{i}}$ é o diâmetro inicial e $\mathrm{L}_{\mathrm{f}}$ diâmetro final de cada corpo de prova.

\section{RESULTADOS E DISCUSSÃO}

$\mathrm{Na}$ caracterização das amostras de argila foram encontrados os seguintes resultados: coloração amarelo queimado, densidade de $0,985 \pm 0,005$ g.mL ${ }^{-1}, \mathrm{pH}$ de 5,46 $\pm 0,05$, umidade de $4,63 \% \pm 0,35 \%$, matéria orgânica de $6,08 \% \pm 0,25 \%$, cinzas de $89,84 \% \pm 0,40 \%$ e quantidade de cromo de $0,05 \% \pm 0,01 \%$. A argila é composta de diferentes espécies mineralógicas, que interferem em suas propriedades físico-químicas e mecânicas. Os resultados da análise térmica e análise infravermelha da argila utilizada podem ser observados nas Figs. 1 e 2, respectivamente. As argilas abrangem um grupo de minerais que possuem uma mesma caracterização, porém pode haver intemperismo, uma vez que sua maior parte de materiais é de origem natural [18]. Em relação à análise térmica da argila, a amostra apresentou perda de massa logo no início do aquecimento (até $250^{\circ} \mathrm{C}$ ) referente à perda de água livre (endotérmico), manteve-se estável até $400{ }^{\circ} \mathrm{C}$ (eliminação da água coloidal da argila) e uma perda acentuada $\left(\sim 500^{\circ} \mathrm{C}\right)$ correspondente à combustão da matéria orgânica e a perda de água por desidroxilação, que é característico de argilominerais, como a caulinita [19]. Esse processo de decomposição, seguido da liberação de água observada, é considerado como sendo irreversível. Nas argilas com pouca quantidade de ferro, a perda de massa ocorre próximo a $700{ }^{\circ} \mathrm{C}$, pois o número de coordenação de $\mathrm{Al}^{3+}$ varia de 6 para 5 [20]. Assim, observouse que a amostra de argila se manteve praticamente estável, até a temperatura de $900{ }^{\circ} \mathrm{C}$, tendo uma perda de massa total de $8,35 \%$. Isto indicou que a amostra utilizada neste estudo apresentou uma quantidade de ferro na sua composição.

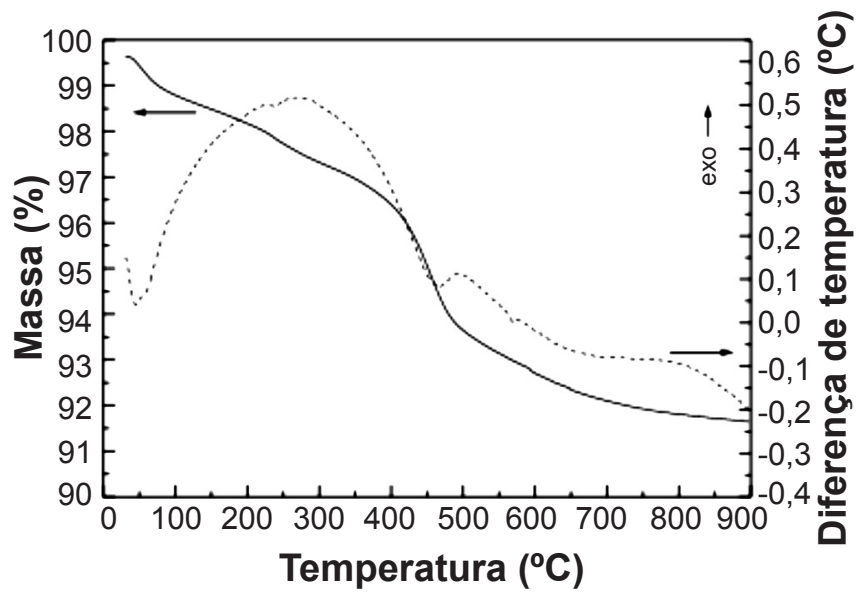

Figura 1: Curvas de TG e DTA da amostra de argila.

[Figure 1: TG and DTA curves of clay sample.] 


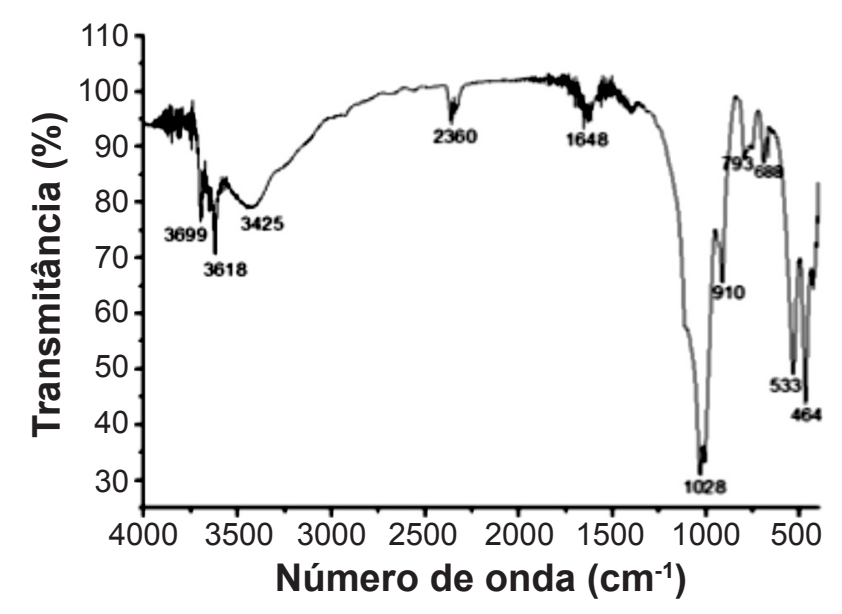

Figura 2: Espectro de IVTF referente à amostra de argila. [Figure 2: FTIR spectrum of clay sample.]

A argila é basicamente um silicato de alumínio hidratado, composta de óxido de alumínio, sílica e água. Junto a estes podem ser encontrados outros compostos, outros minerais, dependendo da região de onde a argila foi retirada. No espectro de IVTF (Fig. 2), o pico de $3699 \mathrm{~cm}^{-1}$ pode ser referente a hidrocarboneto; já os outros picos podem ser referentes a minerais e outros componentes encontrados na argila. As interações das ligações químicas com a radiação infravermelha podem estar associadas aos cátions (ligação $\left.\mathrm{OH}^{-}\right)$, o que pode fornecer informações estruturais da argila $[18,21]$. A banda em $3618 \mathrm{~cm}^{-1}$ foi atribuída mais especificamente ao estiramento $\mathrm{Al}_{2}-\mathrm{OH}$. O pico em 3425 $\mathrm{cm}^{-1}$ pode ser atribuído ao estiramento $\mathrm{Fe}-\mathrm{Al}-\mathrm{OH}$, porém este estiramento ainda não está bem definido na literatura [18]. A banda intensa em $1038 \mathrm{~cm}^{-1}$ foi associada ao estiramento $\mathrm{Si}-\mathrm{O}$ e a $464 \mathrm{~cm}^{-1}$ pode ser associada à deformação $\mathrm{Si}-\mathrm{OH}$ [22, 23]. A banda em $910 \mathrm{~cm}^{-1}$ correspondeu à deformação angular $\mathrm{Al}_{2} \mathrm{OH}$, mesmo sendo com pouca intensidade,

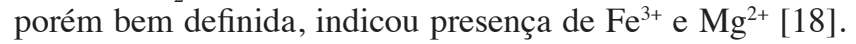
Em $793 \mathrm{~cm}^{-1}$, tem-se uma banda que pode ser associada a uma interação $\mathrm{Al}-\mathrm{Mg}-\mathrm{OH}$, a qual também pode ser atribuída à mistura dos minerais ilita-bentonita $\left(750-960 \mathrm{~cm}^{-1}\right)$ [24] .

$\mathrm{Na}$ caracterização das amostras do lodo galvânico foram encontrados os seguintes resultados: coloração verde musgo, densidade de $0,983 \pm 0,005 \mathrm{~g} \cdot \mathrm{mL}^{-1}, \mathrm{pH}$ de $8,72 \pm 0,04$, umidade de $82,2 \% \pm 1,5 \%$, matéria orgânica de $34,1 \% \pm 0,4 \%$, cinzas de $65,9 \% \pm 0,4 \%$ e quantidade de cromo de $26,06 \% \pm$ $0,02 \%$, valores próximos aos observados em outros estudos [25-27]. As curvas obtidas por análise térmica e análise por infravermelho do lodo utilizado podem ser observadas nas Figs. 3 e 4, respectivamente. Em relação à análise térmica, o lodo com massa inicial de $14,985 \mathrm{mg}$ apresentou perda acentuada de massa desde o início do aquecimento até aproximadamente $200{ }^{\circ} \mathrm{C}$, podendo estar associada à perda de água superficial das partículas de resíduo. Observouse uma perda acentuada em aproximadamente $380{ }^{\circ} \mathrm{C}$, provavelmente relacionada à perda de matéria orgânica com liberação de $\mathrm{H}_{2} \mathrm{O}$ e $\mathrm{CO}_{2}$. Em $550{ }^{\circ} \mathrm{C}$, o pico endotérmico pode estar associado ao processo de perda de carbono inorgânico volátil, presente em carbonatos. Segundo [19], nesta acentuada perda provavelmente ocorreu perda de água higroscópica das partículas do lodo, reduzindo a massa de forma muito acentuada até aproximadamente $650{ }^{\circ} \mathrm{C}$, onde ocorreu provavelmente a combustão e volatilização da matéria orgânica. Em seguida manteve-se estável em 10,299 mg até a temperatura de $900{ }^{\circ} \mathrm{C}$, com perda da massa total de $31,27 \%$.

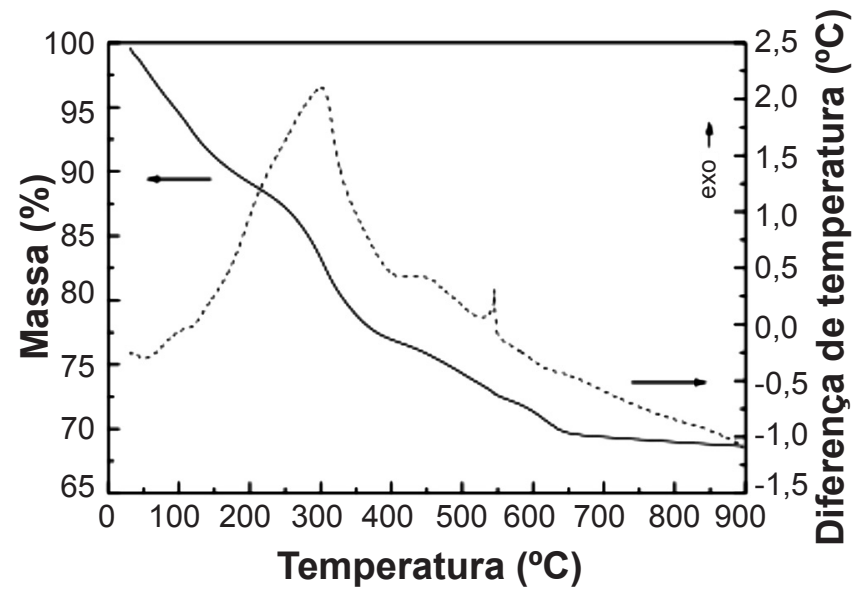

Figura 3: Curvas de TG e DTA da amostra de lodo galvânico. [Figure 3: TG and DTA curves of galvanic sludge sample.]

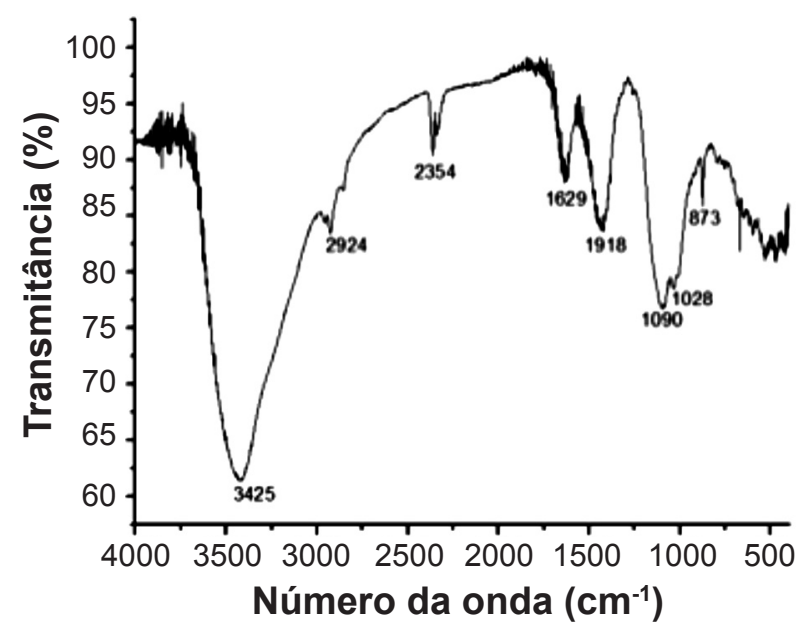

Figura 4: Espectro de IVTF referente à amostra de lodo galvânico. [Figure 4: FTIR spectrum of galvanic sludge sample.]

No lodo de galvanoplastia podem-se encontrar diversos compostos, tais como, hidróxido de sódio, ácido clorídrico, ácido sulfúrico, carbonato de sódio, ácido crômico, níquel e cromo metálico, sais dos metais utilizados, entre outros; estes compostos dependem da empresa e do produto que ela oferta no comércio. No espectro de IVTF (Fig. 4), o pico intenso em $3425 \mathrm{~cm}^{-1}$ refere-se ao grupamento de $\mathrm{O}-\mathrm{H}$; este é encontrado em grande intensidade porque os hidróxidos são utilizados no resíduo para que ocorra a precipitação dos hidróxidos dos metais utilizados no processo. As bandas em $2354 \mathrm{e} 1629 \mathrm{~cm}^{-1}$ podem estar ligadas à presença de água e ao estiramento $\mathrm{C}=\mathrm{O}$, respectivamente. As bandas 


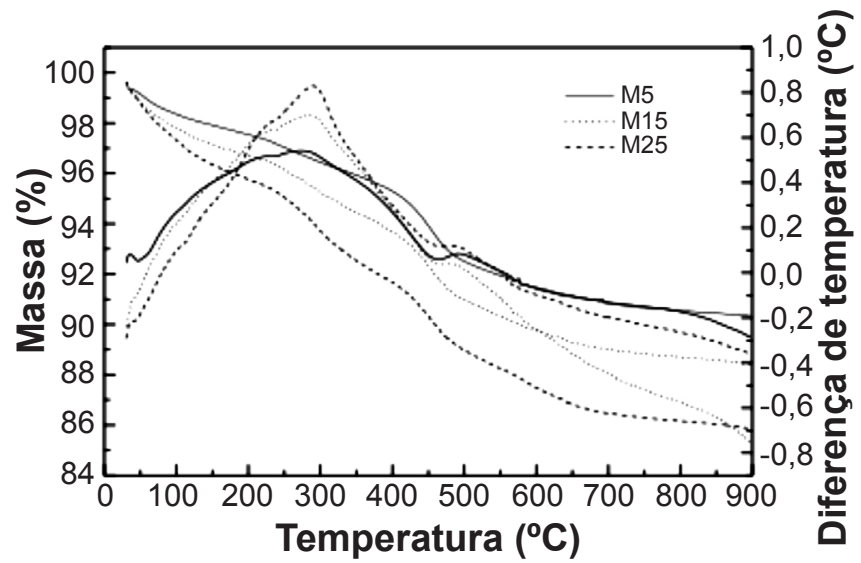

Figura 5: Curvas de TG e DTA das proporções de lodo nas misturas com argila: (a) M5 - 5\% de lodo, (b) M15 - 15\% de lodo e (c) M25 - $25 \%$ de lodo.

[Figure 5: TG and DTA curves of sludge proportions in mixtures with clay: (a) M5 - 5\% of sludge, (b) M15 - 15\% of sludge and (c) M25 - 25\% of sludge.]

entre 1100 e $830 \mathrm{~cm}^{-1}$ podem ser atribuídas a Si-O, muitas vezes apresentando uma contaminação no lodo por silício [28].

As curvas de análise termogravimétrica (TG) e análise térmica diferencial (DTA) referentes às misturas argila/lodo em proporções diferente (lodo: 5, 15 e 25\%) são apresentadas na Fig. 5. A proporção 5\% de lodo em argila teve as mesmas características que a argila pura. Ocorreu uma perda de massa logo no início do aquecimento até aproximadamente $250{ }^{\circ} \mathrm{C}$, associada a um pico endotérmico, que foi referente à perda de água livre, manteve-se praticamente estável até $400{ }^{\circ} \mathrm{C}$, ocorrendo a eliminação da água coloidal da argila, reduzindo sua massa de forma acentuada até aproximadamente $500{ }^{\circ} \mathrm{C}$, que foi referente à perda de água ligada à molécula e à matéria orgânica presente, associada a um pico endotérmico, mantendo-se praticamente estável até o final do processo $\left(900{ }^{\circ} \mathrm{C}\right)$. Nas proporções de 15 e $25 \%$ também ocorreram estas três etapas, mas estas variaram um pouco na escala de temperatura da proporção de $5 \%$, pois apresentaram maior quantidade de matéria orgânica em sua composição. Isso pôde ser observado através da perda de massa maior conforme a proporção de lodo aumentou: a massa inicial da proporção 5\% de lodo foi de 13,048 mg e até o final do tratamento térmico ocorreu uma perda de $9,65 \%$; a massa inicial da proporção $15 \%$ de lodo foi de $14,687 \mathrm{mg}$ com perda total de $11,56 \%$ e a massa inicial da proporção de $25 \%$ foi de $15,045 \mathrm{mg}$ com perda de $14,13 \%$ de massa até o final do tratamento térmico.

Após a preparação de acordo com os parâmetros estabelecidos pelo planejamento experimental, os corpos de prova foram analisados conformes as Normas Brasileiras para cerâmicos. Na Fig. 6 é apresentada a análise de absorção de água para os corpos de prova com misturas argila/lodo. Conforme a NBR 15270-1 [29], a qual diz que "a absorção de água não deve ser inferior a $8 \%$ nem superior a $22 \%$ ", os corpos de prova $3 \mathrm{AB}, 3 \mathrm{CD}$ e $4 \mathrm{CD}$ ultrapassaram o limite máximo de umidade permitida, e o corpo de prova $2 \mathrm{AB}$ não chegou ao valor mínimo permitido. Houve uma redução de absorção de água nos corpos de prova em que a temperatura de queima foi maior $\left(1100{ }^{\circ} \mathrm{C}\right)$, o mesmo observado em estudos com matriz cerâmica e lodo galvânico [30]. Observou-se que as amostras incorporadas com 5\% e 15\% de lodo na argila apresentaram índices de absorção de água dentro dos limites recomendados pela norma, com exceção do corpo de prova $2 \mathrm{AB}$. Esta exceção pode ter ocorrido devido à realização de prensagem deste corpo de prova ou à utilização de quantidade de água a mais que o necessário para fazer a sua mistura, gerando assim maior porosidade em sua massa. Já os corpos de prova contendo $25 \%$ de lodo na argila apresentaram resultados acima do limite ou no caso do corpo de prova $4 \mathrm{AB}$, quase no limite de absorção de água máxima estabelecido pela NBR 15270-1. Além de ser um parâmetro de classificação dos cerâmicos, a absorção de água influencia diretamente sobre as propriedades do produto, uma delas a resistência mecânica: quanto mais
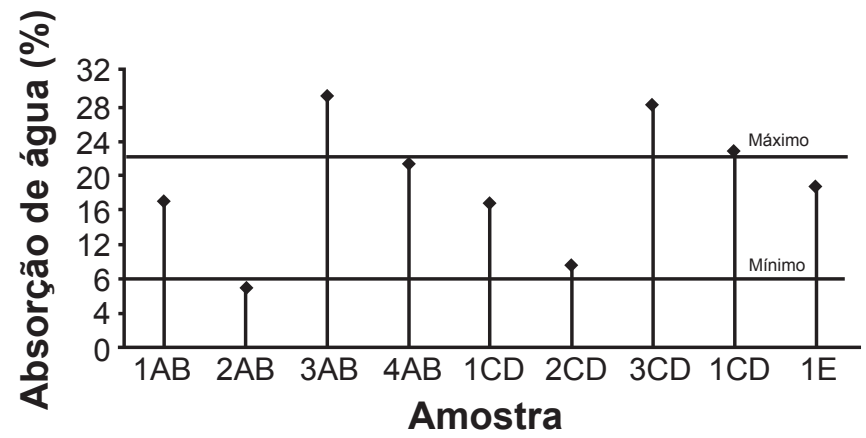

Figura 6: Análise de absorção de água dos corpos de prova e o limite mínimo (8\%) e máximo (22\%) permitido pela NBR 15270-3.

[Figure 6: Water absorption analysis of specimens and the minimum (8\%) and maximum (22\%) values allowed by NBR 15270-3.]

baixa for absorção, maior a resistência mecânica.

$\mathrm{Na}$ Tabela III podem-se observar os valores dos efeitos estimados, coeficientes de regressão, as interações com parâmetros significativos e não significativos, além do valor de p, para a variável dependente, absorção de água. Na análise das estimativas dos efeitos foram considerados os fatores significativos para o intervalo de confiança de $95 \%(\mathrm{p}<0,05)$. Nessa análise de regressão de superfície de resposta todas as variáveis e suas interações foram significativas a $95 \%$ de significância, indicando que $99,88 \%$ da variabilidade dos dados podem ser explicadas pelo modelo. Pôde-se observar que quanto maior a temperatura de secagem, menor a temperatura de queima e maior a concentração de lodo na amostra, maior foi a absorção de água. Nas Figs. 7a, 7b e 7c encontram-se as linhas de contorno para o planejamento fatorial $2^{3}$ para as variáveis concentração de lodo versus temperatura de secagem, concentração de lodo versus temperatura de queima e temperatura de secagem versus temperatura de queima, respectivamente.

As Equações $\mathrm{G}$ a I apresentam as expressões matemáticas da absorção de água nos corpos de prova (AA) em função da concentração de lodo na mistura $\left(\mathrm{X}_{1}\right)$, temperatura de secagem $\left(\mathrm{X}_{2}\right)$ e temperatura de queima $\left(\mathrm{X}_{3}\right)$, fixando-se a 
Tabela III - Análise de regressão dos efeitos, coeficientes de regressão e interações para a variável resposta de absorção de água nos corpos de prova.

[Table III - Effects regression analysis, regression coefficients and interactions for the variable response of water absorption in the specimens.]

\begin{tabular}{cccccc}
\hline Variável & $\begin{array}{c}\text { Soma dos } \\
\text { quadrados }\end{array}$ & $\begin{array}{c}\text { Grau de } \\
\text { liberdade }\end{array}$ & $\begin{array}{c}\text { Média dos } \\
\text { quadrados }\end{array}$ & $\begin{array}{c}\text { Fator de } \\
\text { Student }\end{array}$ & p-valor \\
\hline Concentração $\left(\mathrm{X}_{1}\right)$ & 1,1858 & 1 & 1,1858 & 89,75 & $0,00069^{*}$ \\
T. de secagem $\left(\mathrm{X}_{2}\right)$ & 327,1682 & 1 & 327,1682 & 24762,02 & $0,00000^{*}$ \\
T. de queima $\left(\mathrm{X}_{3}\right)$ & 113,5525 & 1 & 113,5525 & 8594,32 & $0,00000^{*}$ \\
$\left(\mathrm{X}_{1}\right) \mathrm{X}\left(\mathrm{X}_{2}\right)$ & 0,5000 & 1 & 0,5000 & 37,84 & $0,00354^{*}$ \\
$\left(\mathrm{X}_{1}\right) \mathrm{X}\left(\mathrm{X}_{3}\right)$ & 3,4061 & 1 & 3,4061 & 257,79 & $0,00008^{*}$ \\
$\left(\mathrm{X}_{2}\right) \mathrm{X}\left(\mathrm{X}_{3}\right)$ & 2,1840 & 1 & 2,1840 & 165,30 & $0,00021^{*}$ \\
Erro & 0,0529 & 4 & 0,0132 & & \\
Total SS & 448,0494 & 10 & & & \\
\hline
\end{tabular}

* - Variáveis e interações significativas.
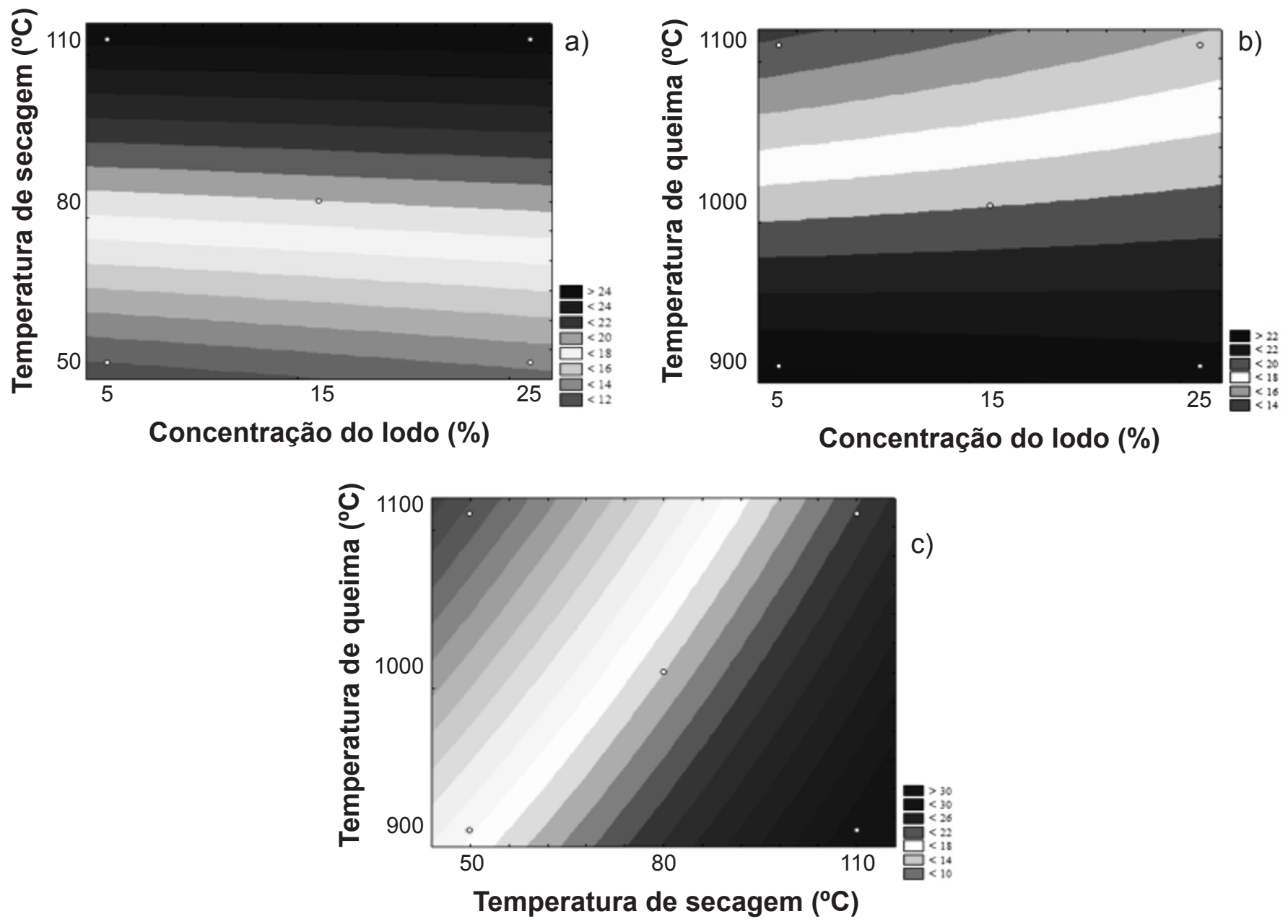

Figura 7: Linhas de contorno de absorção de água (\%) para planejamento fatorial $2^{3}$ : (a) concentração de lodo versus temperatura de secagem; (b) concentração de lodo versus temperatura de queima; (c) temperatura de secagem versus temperatura de queima.

[Figure 7: Water absorption (\%) contour lines for $2^{3}$ factorial design: (a) sludge concentration versus drying temperature; (b) sludge concentration versus firing temperature; (c) drying temperature versus firing temperature.]

variável temperatura de queima $\left(\mathrm{X}_{3}\right)$ ao nível zero $\left(1000^{\circ} \mathrm{C}\right)$ na Equação $\mathrm{G}$, temperatura de secagem $\left(\mathrm{X}_{2}\right)$ ao nível zero $\left(80{ }^{\circ} \mathrm{C}\right)$ na Equação $\mathrm{H}$ e concentração de lodo na mistura
$\left(\mathrm{X}_{1}\right)$ ao nível zero $(15 \%)$ na Equação I. Para o cerâmico com incorporação de lodo galvânico estar dentro dos limites estabelecidos para o parâmetro absorção de água, o processo 
Tabela IV - Análise de regressão dos efeitos, coeficientes de regressão e interações para a variável resposta de lixiviação de cromo nos corpos de prova.

[Table IV - Effects regression analysis, regression coefficients and interactions for the variable response of chromium leaching in the specimens.]

\begin{tabular}{cccccc}
\hline Variável & $\begin{array}{c}\text { Soma dos } \\
\text { quadrados }\end{array}$ & $\begin{array}{c}\text { Grau de } \\
\text { liberdade }\end{array}$ & $\begin{array}{c}\text { Média dos } \\
\text { quadrados }\end{array}$ & $\begin{array}{c}\text { Fator de } \\
\text { Student }\end{array}$ & p-valor \\
\hline Concentração $\left(\mathrm{X}_{1}\right)$ & 0,190653 & 1 & 0,190653 & 9,71826 & $0,03561^{*}$ \\
T. de secagem $\left(\mathrm{X}_{2}\right)$ & 0,002628 & 1 & 0,002628 & 0,13396 & 0,73289 \\
T. de queima $\left(\mathrm{X}_{3}\right)$ & 0,081003 & 1 & 0,081003 & 4,12901 & 0,11195 \\
$\left(\mathrm{X}_{1}\right) \mathrm{X}\left(\mathrm{X}_{2}\right)$ & 0,261003 & 1 & 0,261003 & 13,30424 & $0,02182^{*}$ \\
$\left(\mathrm{X}_{1}\right) \mathrm{X}\left(\mathrm{X}_{3}\right)$ & 0,013203 & 1 & 0,013203 & 0,67301 & 0,45807 \\
$\left(\mathrm{X}_{2}\right) \mathrm{X}\left(\mathrm{X}_{3}\right)$ & 0,102378 & 1 & 0,102378 & 5,21857 & 0,08437 \\
Erro & 0,078472 & 4 & 0,019618 & & \\
Total SS & 0,729341 & 10 & & & \\
\hline
\end{tabular}

* - Variáveis e interações significativas.

de fabricação deste produto, conforme o planejamento experimental, deve ter incorporação abaixo de $15 \%$, temperatura de secagem máxima de $80{ }^{\circ} \mathrm{C}$ e temperatura de queima alta $\left(1100^{\circ} \mathrm{C}\right)$.

$$
\begin{aligned}
& \text { AA }(\%)=18,98+0,38 * X_{1}+6,40 * X_{2}-0,25 * X_{1} * X_{2} \\
& \text { AA }(\%)=18,98+0,38 * X_{1}-3,77 * X_{3}-0,65 * X_{1} * X_{3} \\
& \text { AA }(\%)=18,98+6,40 * X_{2}-3,77 * X_{3}-0,52 * X_{2} * X_{3}
\end{aligned}
$$

Os resultados obtidos na lixiviação das amostras e o limite máximo permitido pela NBR 10004 estão apresentados na Fig. 8. Os resultados obtidos na leitura de cromo nas soluções dos corpos de prova lixiviados se apresentaram na faixa de $0,0950 \pm 0,0070$ a $0,8800 \pm 0,0014 \mathrm{mg} \cdot \mathrm{L}^{-1}$, todos dentro do limite máximo (5 mg. $\mathrm{L}^{-1}$ de $\mathrm{Cr}$ ) permitido [3]. Na Tabela IV podem-se observar os valores dos efeitos estimados, coeficientes de regressão, as interações com parâmetros significativos e não significativos, além do valor de p, para a variável dependente, lixiviação de cromo. Na análise das estimativas dos efeitos foram considerados os fatores significativos para o intervalo de confiança de $95 \%$ $(p<0,05)$. Nessa análise de regressão de superfície de resposta a variável dependente concentração de lodo e sua interação com a temperatura de secagem foram significativas a $95 \%$ de significância, indicando que $89,24 \%$ da variabilidade dos dados podem ser explicadas pelo modelo. Na Fig. 9 encontram-se as linhas de contorno para o planejamento fatorial $2^{3}$ para as variáveis concentração de lodo versus temperatura de secagem.

A Equação $\mathrm{J}$ apresenta a expressão matemática da concentração de lodo na mistura $\left(X_{1}\right)$ e temperatura de secagem $\left(\mathrm{X}_{2}\right)$ sobre a lixiviação de cromo dos corpos de prova (LC), fixando-se a variável temperatura de queima $\left(\mathrm{X}_{3}\right)$ ao nível zero $\left(1000^{\circ} \mathrm{C}\right)$. Observou-se novamente que corpos de prova com temperatura de secagem abaixo de $80^{\circ} \mathrm{C}$ continuou apresentando uma melhor tendência para os parâmetros das normas. Em se tratando da concentração de lodo na mistura, para este parâmetro, mesmo sendo significante estatisticamente, observa-se pela Fig. 8 que a faixa de concentração de cromo na mistura estudada neste

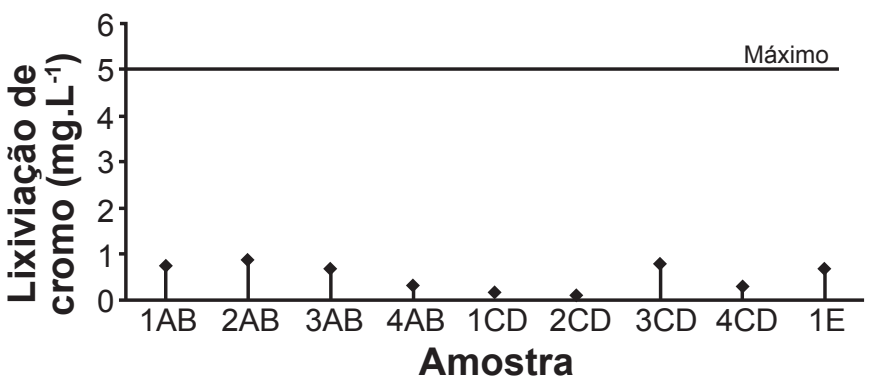

Figura 8: Resultados do teste de lixiviação de cromo dos corpos de prova e o limite máximo (5 mg.L $\left.\mathrm{L}^{-1}\right)$ permitido pela NBR 10004. [Figure 8: Results of chromium leaching test of specimens and the maximum limit (5 $\left.\mathrm{mg} . \mathrm{L}^{-1}\right)$ according to NBR 10004.]

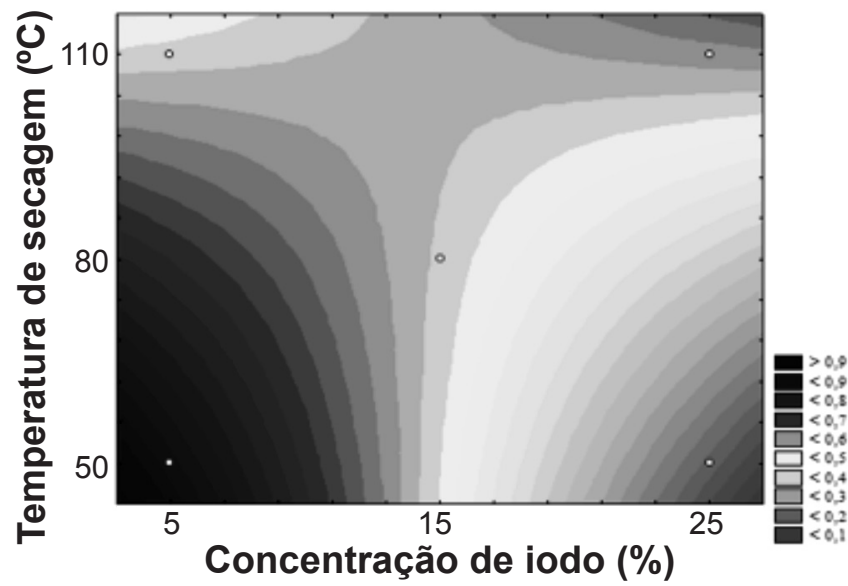

Figura 9: Linhas de contorno de concentração de cromo lixiviado (mg. $\mathrm{L}^{-1}$ ) para planejamento fatorial $2^{3}$ : concentração de lodo versus temperatura de secagem.

[Figure 9: Chromium concentration $\left(m g . L^{-1}\right)$ contour lines for $2^{3}$ factorial design: sludge concentration versus drying temperature.] 
Tabela V - Análise de regressão dos efeitos, coeficientes de regressão e interações para a variável resposta da solubilização de cromo nos corpos de prova.

[Table $V$ - Effects regression analysis, regression coefficients and interactions for the variable response of chromium solution in the specimens.]

\begin{tabular}{cccccc}
\hline Variável & $\begin{array}{c}\text { Soma dos } \\
\text { quadrados }\end{array}$ & $\begin{array}{c}\text { Grau de } \\
\text { liberdade }\end{array}$ & $\begin{array}{c}\text { Média dos } \\
\text { quadrados }\end{array}$ & $\begin{array}{c}\text { Fator de } \\
\text { Student }\end{array}$ & p-valor \\
\hline Concentração $\left(\mathrm{X}_{1}\right)$ & 0,01424 & 1 & 0,014238 & 0,009276 & 0,92791 \\
T. de secagem $\left(\mathrm{X}_{2}\right)$ & 3,49471 & 1 & 3,494707 & 2,276634 & 0,20584 \\
T. de queima $\left(\mathrm{X}_{3}\right)$ & 4,88297 & 1 & 4,882969 & 3,181020 & 0,14907 \\
$\left(\mathrm{X}_{1}\right) \mathrm{X}\left(\mathrm{X}_{2}\right)$ & 0,07752 & 1 & 0,077520 & 0,050500 & 0,83321 \\
$\left(\mathrm{X}_{1}\right) \mathrm{X}\left(\mathrm{X}_{3}\right)$ & 0,01999 & 1 & 0,019990 & 0,013023 & 0,91464 \\
$\left(\mathrm{X}_{2}\right) \mathrm{X}\left(\mathrm{X}_{3}\right)$ & 1,30016 & 1 & 1,300159 & 0,846991 & 0,40949 \\
Erro & 6,14013 & 4 & 1,535032 & & \\
Total SS & 15,92971 & 10 & & & \\
\hline
\end{tabular}

* - Variáveis e interações significativas.

trabalho apresentou-se dentro da legislação estabelecida para lançamento de efluentes - $\mathrm{Cr}^{3+}: 1 \mathrm{mg} . \mathrm{L}^{-1}[31]$.

$\mathrm{LC}\left(\mathrm{mg} \cdot \mathrm{L}^{-1}\right)=0,54-0,15 * \mathrm{X}_{1}+0,22 * \mathrm{X}_{2}-0,18 * \mathrm{X}_{1} * \mathrm{X}_{2}(\mathrm{~J})$

Os resultados obtidos na solubilização das amostras e o limite máximo permitido pela NBR 10004 estão apresentados na Fig. 10. Para a leitura de cromo das soluções dos corpos de prova na análise de solubilização foram observados valores na faixa $0,2625 \pm 0,0028$ a $3,2250 \pm 0,0028 \mathrm{mg} \cdot \mathrm{L}^{-1}$; todos esses valores ultrapassaram o valor estabelecido (0,05 mg Cr L $\left.{ }^{-1}\right)$ na norma NBR 10004 [3], determinando sua classificação como classe II - A (não inerte), o que difere dos estudos [32,33], que apresentaram inertização do cromo na matriz cerâmica, porém os mesmos trabalharam com até 5\% de lodo incorporado na cerâmica vermelha. Na Tabela V podem-se observar os valores dos efeitos estimados, coeficientes de regressão, as interações com parâmetros significativos e não significativos, além do valor de p, para a variável dependente, solubilização de cromo. Na análise das estimativas dos efeitos foram considerados os fatores significativos para o intervalo de confiança de $95 \%$ $(p<0,05)$. Nessa análise, todas as variáveis e suas interações não foram significativas a 95\%, não tendo influência sobre

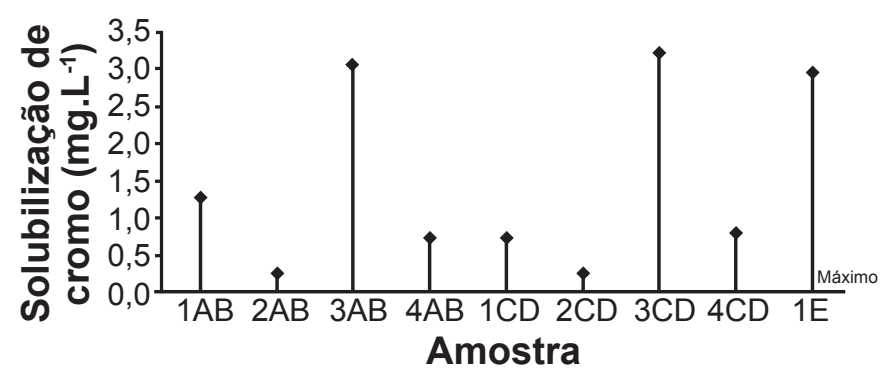

Figura 10: Resultados do teste de solubilização de cromo nas amostras e seu limite máximo $\left(0,05\right.$ mg.L $\left.\mathrm{L}^{-1}\right)$ segundo a NBR 10004. [Figure 10: Results of chromium solution test and the maximum limit (0.05 $\left.\mathrm{mg} . \mathrm{L}^{-1}\right)$ according to NBR 10004.] a solubilização de resíduos. Porém, avaliando a Fig. 10, observam-se valores menores para os corpos de prova que foram submetidos à temperatura de queima de $1100{ }^{\circ} \mathrm{C}$. Essa redução poderia ser efeito da sinterização do material no processo da queima.

Nas Tabelas VI e VII encontram-se os resultados da retração linear nas amostras e os valores dos efeitos estimados, coeficientes de regressão, as interações com parâmetros significativos e não significativos, além do valor de $\mathrm{p}$, para a variável dependente, retração linear dos corpos de prova, respectivamente. As retrações lineares obtidas nos corpos de prova se apresentaram na faixa de 7,89\% $\pm 0,55 \%$ a $21,26 \% \pm 10,34 \%$. Observou-se que a retração dos corpos de prova entre sua moldagem e sua queima foi bastante variável, porém os maiores valores foram para temperatura de queima maior $\left(1100{ }^{\circ} \mathrm{C}\right)$, também observado em estudos na literatura [30]. Isso pode ter ocorrido devido ao excesso ou falta de água inserida na mistura para que esta se tornasse homogênea, resultando assim em uma maior ou menor retração linear nos corpos. Observou-se também que os corpos de prova com a mesma proporção de lodo em sua massa e a mesma temperatura de queima possuíram retrações

Tabela VI - Resultados da retração linear nas amostras. [Table VI - Results of linear shrinkage of samples.]

\begin{tabular}{cc}
\hline Identificação & $\%$ de retração \\
\hline $1 \mathrm{AB}$ & $14,48 \pm 3,94$ \\
$2 \mathrm{AB}$ & $19,85 \pm 6,26$ \\
$3 \mathrm{AB}$ & $8,88 \pm 0,03$ \\
$4 \mathrm{AB}$ & $18,52 \pm 2,86$ \\
$1 \mathrm{CD}$ & $14,21 \pm 0,37$ \\
$2 \mathrm{CD}$ & $18,27 \pm 6,02$ \\
$3 \mathrm{CD}$ & $7,89 \pm 0,55$ \\
$4 \mathrm{CD}$ & $10,93 \pm 8,12$ \\
$1 \mathrm{E}$ & $21,26 \pm 10,34$ \\
\hline
\end{tabular}


Tabela VII - Análise de regressão dos efeitos, coeficientes de regressão e interações para a variável resposta da retração linear dos corpos de prova.

[Table VII - Effects regression analysis, regression coefficients and interactions for the variable response of linear shrinkage in the specimens.]

\begin{tabular}{cccccc}
\hline Variável & $\begin{array}{c}\text { Soma dos } \\
\text { quadrados }\end{array}$ & $\begin{array}{c}\text { Grau de } \\
\text { liberdade }\end{array}$ & $\begin{array}{c}\text { Média dos } \\
\text { quadrados }\end{array}$ & $\begin{array}{c}\text { Fator de } \\
\text { Student }\end{array}$ & p-valor \\
\hline Concentração $\left(\mathrm{X}_{1}\right)$ & 13,5981 & 1 & 13,59811 & 0,475235 & 0,52849 \\
T. de secagem $\left(\mathrm{X}_{2}\right)$ & 52,9935 & 1 & 52,99351 & 1,852049 & 0,24517 \\
T. de queima $\left(\mathrm{X}_{3}\right)$ & 61,1065 & 1 & 61,10651 & 2,135587 & 0,21772 \\
$\left(\mathrm{X}_{1}\right) \mathrm{X}\left(\mathrm{X}_{2}\right)$ & 5,6616 & 1 & 5,66161 & 0,197865 & 0,67946 \\
$\left(\mathrm{X}_{1}\right) \mathrm{X}\left(\mathrm{X}_{3}\right)$ & 7,8210 & 1 & 7,82101 & 0,273333 & 0,62873 \\
$\left(\mathrm{X}_{2}\right) \mathrm{X}\left(\mathrm{X}_{3}\right)$ & 1,3203 & 1 & 1,32031 & 0,046143 & 0,84042 \\
Erro & 114,4538 & 4 & 28,61344 & & \\
Total SS & 256,9549 & 10 & & & \\
\hline
\end{tabular}

* - Variáveis e interações significativas.

lineares similares umas com as outras, com exceção do $4 \mathrm{AB}$ e $4 \mathrm{CD}$. Porém na análise de regressão de superfície de resposta todas as variáveis e suas interações não foram significativas a 95\% de significância, ou seja, nenhuma das variáveis estudadas teve influência sobre a retração linear dos corpos de prova.

\section{CONCLUSÕES}

Analisando os resultados obtidos nas caracterizações dos corpos de prova verificou-se a possibilidade de desenvolver cerâmicos incorporados com lodo galvânico. Os resultados de absorção de água e retração linear indicaram que nas faixas dos parâmetros investigados os corpos de provas produzidos com temperatura de queima de $1100{ }^{\circ} \mathrm{C}$ apresentaram valores que possibilitaram enquadrar os materiais cerâmicos produzidos com essa formulação para aplicações como telhas e blocos cerâmicos. Quanto à imobilização de cromo presente no lodo galvânico, não houve viabilidade técnica na incorporação do lodo em matriz cerâmica nas condições propostas no planejamento experimental e, assim, classificaram-se como resíduos não inertes (Classe II - A).

\section{REFERÊNCIAS}

[1] T.C.F. do Nascimento, C.H. Mothé, Rev. Anal. 27 (2007) 36.

[2] L.C. Alves, E.S.M. Seo, Eng. Sanitária Ambiental 19, 4 (2014) 423.

[3] ABNT - Associação Brasileira de Normas Técnicas, NBR 10004, "Resíduos sólidos: classificação", Rio de Janeiro, RJ (2004).

[4] V.E. Schneider, F. Bettin, F. Parise Jr., Anais do XXVII Congresso Interamericano de Engenharia Sanitária e Ambiental, Porto Alegre, RS (2000) 1.

[5] F. Garrido, A. Gentil, L Thomé, Surf. Coat. Technol. 196 (2005) 63.

[6] C.R. Cheeseman, S.M. Rocha, C. Sollars, S. Bethanis,
A.R. Boccaccini, Waste Manage. 23 (2003) 907.

[7] I.T. Kim, J.H. Kim, K.S. Lee, Y.C. Seo, J.K. Koo, Waste Manage. 20 (2000) 409.

[8] M.R.D. Romero, J.M.A. Rawlings, J. Eur. Ceram. Soc. 19 (1999) 2049.

[9] A.C. Silva, S.R.H.M. Castanho, J. Non-Cryst. Solids 348 (2004) 211.

[10] M. Pelino, Waste Manage. 20 (2000) 561.

[11] S.N. Monteiro, J. Alexandre, J.I. Margem, R. Sánchez, C.M.F. Vieira, Construc. Buil. Mater. 22 (2008) 1281.

[12] American Public Health Association, Standard methods for the examination for water and wasterwater, $19^{\text {th }}$ Ed., Washington (1995).

[13] ABNT - Associação Brasileira de Normas Técnicas, NBR 13738, "Determinação de cromo hexavalente - método colorimétrico da difenilcarbazida", Rio de Janeiro, RJ (1996).

[14] ABNT - Associação Brasileira de Normas Técnicas, NBR 15270-3, "Blocos cerâmicos para alvenaria estrutural e de vedação - métodos de ensaio", Rio de Janeiro, RJ (2005).

[15] ABNT - Associação Brasileira de Normas Técnicas, NBR 10005, "Lixiviação de resíduos", Rio de Janeiro, RJ (1987).

[16] ABNT - Associação Brasileira de Normas Técnicas, NBR 10006, "Solubilização de resíduos", Rio de Janeiro, RJ (1987).

[17] ABNT - Associação Brasileira de Normas Técnicas, NBR MB 305, "Determinação da retração linear", Rio de Janeiro, (1984).

[18] F.Q. Mariani, J.C. Villalba, F.J. Anaissi, Orbital 5, 4 (2013) 249.

[19] O.T. Kaminata, C.R.G. Tavares, C.T. Benatti, F.M.S. Ramos, M. Capelasso, Cerâmica Ind. 13, 3 (2008) 39.

[20] A. Meunier, Clays, $1^{\text {st }}$ Ed., Springer Verlag, Germany (2005) 472.

[21] L.G. Lima Jr., C.W. Frederico, M.V.S. Fernandes, A.R. Loiola, J.C.R.A. Andrade, C.P. Moura, I.C. Mattos, J.A. Nogueira Neto, L.R.D. Silva, Geochim. Brasiliensis 25, 1 (2011) 7.

[22] R.M. Silverstein, G.C. Bassler, T.C. Morrill, Spectrometric identification of organic compounds, $5^{\text {th }}$ Ed., John Wiley \& 
Sons, New York (1991) 430.

[23] F. Rouquerol, J. Rouquerol, P. Llwellyn, Thermal analysis. Handbook of clay science, $1^{\mathrm{st}}$ Ed., Elsevier Science, China (2006) 1246.

[24] V.K. Pecharsky, P.Y. Zavalij, Fundamentals of powder diffraction and structural characterization of materials, $2^{\text {nd }}$ Ed., Springer, New York (2009) 741.

[25] T.M. Basegio, D.L. Villanova, C.P. Bergmann, Anais do Congreso Internacional en Ciencia y Tecnología de Metalurgia y Materiales, San Nicolá, Buenos Aires (2007).

[26] K.W. Milanez, C.T. Kniess, A.M. Bernardin, H.G. Riella, N.C. Kuhnen, Cerâmica 51, 318 (2005) 107.

[27] J. Viguri, A. Andrés, R. Ibañez, C.R. Puente, A. Irabien, J. Hazard. Mater. 79, 1 (2000) 63.

[28] É. Makó, Z. Senkár, J. Kristóf, V. Vágvölgyi, J. Colloid
Interface Sci. 294, 2 (2006) 362.

[29] ABNT - Associação Brasileira de Normas Técnicas, NBR 15270-1, "Componentes cerâmicos. Parte 1: Blocos cerâmicos para alvenaria de vedação - terminologia e requisitos", Rio de Janeiro, RJ (2005).

[30] A.C. Teloeken, D.L. Villanova, T.M. Basegio, C.P. Bergmann, Cerâmica Ind. 16, 2 (2011) 14.

[31] Conselho Nacional do Meio Ambiente, Resolução $n^{\circ}$ 430, de 13 de maio de 2011, "Dispõe sobre as condições e padrões de lançamento de efluentes" (2011).

[32] V.T. Balaton, P.S. Gonçalves, L.M. Ferrer, Cerâmica Ind. 7, 6 (2002) 42 .

[33] J.M. Magalhães, J.E. Silva, F.P. Castro, J .A. Labrincha, J. Hazard. Mater. 106B (2004) 169.

(Rec.05/08/2015, Rev. 21/03/2016, Ac. 22/05/2016) 